Supporting Information for:

\title{
Biosensors for Managing the COVID-19 Cytokine Storm: Challenges Ahead.
}

Steven M. Russell, ${ }^{1}$ Alejandra Alba-Patiño, ${ }^{1}$ Enrique Barón, ${ }^{1}$ Marcio Borges,,${ }^{1,3}$ Marta González-Freire ${ }^{2, *}$ and Roberto de la Rica. ${ }^{1, *}$

${ }^{1}$ Multidisciplinary Sepsis Group and ${ }^{2}$ Vascular and Metabolic Pathologies Group, Health Research Institute of the Balearic Islands (IdISBa), Son Espases University Hospital, S building, Ctra. de Valldemossa 79, 07120 Palma de Mallorca, Spain.

${ }^{3}$ Multidisciplinary Sepsis Unit, ICU, Son Llàtzer University Hospital. 
Table S1. Examples of biosensors for cytokine detection proposed in the last 5 years. Previous examples can be found in Biosensors and Bioelectronics, 2016 79, 810-821 and references therein; iLOD is the limit of detection in ideal conditions (buffered solution), mLOD is the limit of detection in a real matrix.

\begin{tabular}{|c|c|c|c|c|c|c|c|c|c|c|}
\hline REF & technique & Detection & Support & Cytokines & iLOD & iMatrix & $\begin{array}{c}\text { Dynamic } \\
\text { range }\end{array}$ & Sample & mLOD & $\begin{array}{c}\text { Analysis } \\
\text { time }\end{array}$ \\
\hline 1 & Immunosensor & Chemiluminescence & optical fiber & IL-6 & $\begin{array}{c}1.05 \\
\mathrm{pg} \\
\mathrm{mL}^{-1}\end{array}$ & PBS & $\begin{array}{l}5-10000 \\
\mathrm{pg} \mathrm{mL}^{-1}\end{array}$ & $\begin{array}{l}\text { human } \\
\text { serum }\end{array}$ & --- & $>75 \min$ \\
\hline 2 & Immunosensor & $\begin{array}{c}\text { Electrochemical } \\
\text { impedance }\end{array}$ & $\begin{array}{l}\text { indium tin oxide } \\
\text { (ITO) electrode }\end{array}$ & IL-6 & $\begin{array}{c}6.0 \mathrm{fg} \\
\mathrm{mL}^{-1}\end{array}$ & PBS & $\begin{array}{l}0.02-16 \\
\mathrm{pg} \mathrm{mL}^{-1}\end{array}$ & $\begin{array}{l}\text { human } \\
\text { serum }\end{array}$ & --- & $>30 \mathrm{~min}$ \\
\hline 3 & Immunosensor & Colorimetric & $\begin{array}{c}\text { plasmonic } \\
\text { nanoprobes } \\
\text { and paper }\end{array}$ & IL-6 & $\begin{array}{c}0.1 \mathrm{pg} \\
\mathrm{mL}^{-1}\end{array}$ & PBS & --- & $\begin{array}{l}\text { whole } \\
\text { blood }\end{array}$ & --- & $17 \mathrm{~min}$ \\
\hline 4 & Immunosensor & Electrochemical & gold electrode & IL-6 & $\begin{array}{l}4 \mathrm{pg} \\
\mathrm{mL}^{-1}\end{array}$ & PBS & --- & $\begin{array}{l}\text { human } \\
\text { plasma }\end{array}$ & 23 pg/mL & $>110 \min$ \\
\hline 5 & $\begin{array}{l}\text { Oligonucleotides } \\
\text { and antibodies }\end{array}$ & Optical & $\begin{array}{c}\text { Silica wafers coated } \\
\text { with } \mathrm{Ti} / \mathrm{Au}\end{array}$ & IL-6 & $\begin{array}{c}88 \mu \mathrm{g} \\
\mathrm{mL}^{-1}\end{array}$ & $\begin{array}{l}\text { PBS } \\
\text { and } \\
\text { SSC }\end{array}$ & ---- & --- & --- & --- \\
\hline 6 & Immunosensor & $\begin{array}{c}\text { Surface-enhanced } \\
\text { Raman scattering } \\
\text { (SERS) }\end{array}$ & $\begin{array}{l}\text { paper and DTNB } \\
\text { on gold nano shell } \\
\text { with a silica core }\end{array}$ & IL-6 & $\begin{array}{l}1 \mathrm{pg} \\
\mathrm{mL}^{-1}\end{array}$ & PBS & $\begin{array}{l}1 \mathrm{pg} \mathrm{mL}^{-1}- \\
1 \mathrm{\mu g} \mathrm{mL}^{-1}\end{array}$ & $\begin{array}{l}\text { whole } \\
\text { blood }\end{array}$ & $5 \mathrm{pg} / \mathrm{mL}$ & --- \\
\hline 7 & Aptasensor & $\begin{array}{l}\text { Electrochemical } \\
\text { impedance }\end{array}$ & $\begin{array}{l}\text { glassy carbon } \\
\text { electrode } \\
\text { modified with } \\
\text { p-aminobenzoic } \\
\text { acid, } \\
\text { p-aminothiophenol } \\
\text { and AuNPs }\end{array}$ & IL-6 & $\begin{array}{c}1.6 \mathrm{pg} \\
\mathrm{mL}^{-1}\end{array}$ & PBS & $\begin{array}{c}5 \mathrm{pg} \mathrm{mL}^{-1}- \\
100 \mathrm{ng} \\
\mathrm{mL}^{-1}\end{array}$ & $\begin{array}{l}\text { human } \\
\text { serum }\end{array}$ & ---- & $>60 \mathrm{~min}$ \\
\hline 8 & Immunosensor & Electrochemical & $\begin{array}{c}\text { magnetic } \\
\text { microparticles and } \\
\text { planar graphite- } \\
\text { screen } \\
\text { printed electrodes }\end{array}$ & IL-6 & $\begin{array}{c}0.3 \mathrm{pg} \\
\mathrm{mL}^{-1}\end{array}$ & PBS & $\begin{array}{l}1 \mathrm{pg} \mathrm{mL}^{-1}- \\
1 \mathrm{\mu g} \mathrm{mL}^{-1}\end{array}$ & $\begin{array}{l}\text { human } \\
\text { serum }\end{array}$ & --- & $\begin{array}{c}30-60 \\
\min \end{array}$ \\
\hline 9 & Immunosensor & Fluorescence & $\begin{array}{l}\text { Nitrocellulose, PVC, } \\
\text { glass fiber }\end{array}$ & IL-6 & $\begin{array}{c}0.37 \\
\mathrm{pg} \\
\mathrm{mL}^{-1}\end{array}$ & PBST-BSA & $\begin{array}{c}2-500 \mathrm{pg} \\
\mathrm{mL}^{-1}\end{array}$ & $\begin{array}{l}\text { human } \\
\text { serum }\end{array}$ & --- & $15 \min$ \\
\hline
\end{tabular}




\begin{tabular}{|c|c|c|c|c|c|c|c|c|c|c|}
\hline 10 & Immunosensor & $\begin{array}{l}\text { Fluorescence } \\
\text { spectroscopy }\end{array}$ & nitrocellulose strip & IL-6 & $\begin{array}{c}0.9 \mathrm{pg} \\
\mathrm{mL}^{-1}\end{array}$ & PBS & $\begin{array}{c}1-1000 \mathrm{pg} \\
\mathrm{mL}^{-1}\end{array}$ & serum & ---- & $30 \mathrm{~min}$ \\
\hline 11 & $\begin{array}{c}\text { Aptameric } \\
\text { GFET (field } \\
\text { effect transistor } \\
\text { ) nanosensor }\end{array}$ & Electrochemical & graphene & IL-6 & $\begin{array}{c}210 \\
\mathrm{pg} \\
\mathrm{mL}^{-1}\end{array}$ & PBS & $\begin{array}{c}50-800 \\
\text { pM }\end{array}$ & $\begin{array}{l}\text { human } \\
\text { saliva }\end{array}$ & $\begin{array}{l}12.2 \mathrm{pM} \\
(\approx 250 \\
\mathrm{pg} / \mathrm{ml})\end{array}$ & $6 \min$ \\
\hline 12 & Immunosensor & $\begin{array}{l}\text { Differential pulse } \\
\text { voltammetry }\end{array}$ & $\begin{array}{c}\text { TI:Au on silicon } \\
\text { subtrate }\end{array}$ & IL-6 & $\begin{array}{l}20 \mathrm{pg} \\
\mathrm{mL}^{-1}\end{array}$ & PBS & $\begin{array}{c}0-60 \mathrm{pg} \\
\mathrm{mL}^{-1}\end{array}$ & $\begin{array}{l}\text { BSA 5\% } \\
\text { and } \\
\text { human } \\
\text { serum }\end{array}$ & & $2.5 \min$ \\
\hline 13 & Immunosensor & $\begin{array}{c}\text { Localized-surface } \\
\text { plasmon resonance } \\
\text { (LSPR) }\end{array}$ & gold nanorod & $\begin{array}{l}\text { IL-6, TNF- } \\
\alpha, \text { IL-10 } \\
\text { and IL-4 }\end{array}$ & $\begin{array}{l}10 \mathrm{pg} \\
\mathrm{mL}^{-1}\end{array}$ & PBS & $\begin{array}{l}10-10000 \\
\mathrm{pg} \mathrm{mL}^{-1}\end{array}$ & $\begin{array}{c}\text { adipose } \\
\text { tissue cell } \\
\text { culture } \\
\end{array}$ & $20 \mathrm{pg} / \mathrm{ml}$ & $30 \mathrm{~min}$ \\
\hline 14 & Immunosensor & $\begin{array}{c}\text { Electrochemical } \\
\text { (conductance signal) }\end{array}$ & $\begin{array}{c}\text { silicon } \\
\text { nanowire } \\
\text { field effect transistor }\end{array}$ & IL-6 & ----- & PBS & $\begin{array}{l}5-50000 \\
\mathrm{pg} \mathrm{mL}^{-1}\end{array}$ & $\begin{array}{c}\text { rat } \\
\text { exhaled } \\
\text { breath }\end{array}$ & ---- & real-time \\
\hline 15 & Immunosensor & $\begin{array}{l}\text { Fluorescence } \\
\text { spectroscopy }\end{array}$ & optical fibre. & IL-6 & $\begin{array}{c}0.1 \mathrm{pg} \\
\mathrm{mL}^{-1}\end{array}$ & PBS & $\begin{array}{c}0.4 \mathrm{pg} \mathrm{mL}_{1}^{-} \\
\text {to } 400 \mathrm{pg} \\
\mathrm{mL}^{-1}\end{array}$ & $\begin{array}{l}\text { PBS }+10 \% \\
\text { FBS. cell } \\
\text { culture } \\
\text { medium, } \\
\text { from BV-2 } \\
\text { cells. } \\
\end{array}$ & ---- & $60 \mathrm{~min}$ \\
\hline 16 & Immunosensor & $\begin{array}{l}\text { Electrochemical } \\
\text { (amperometric) }\end{array}$ & graphene oxides & $\begin{array}{l}\text { IL-6, } \\
\text { IL-1 } \beta \text {, } \\
\text { and } \\
\text { TNF- } \alpha\end{array}$ & $\begin{array}{l}5 \mathrm{pg} \\
\mathrm{mL}^{-1}\end{array}$ & PBS & $\begin{array}{c}5-150 \mathrm{pg} \\
\mathrm{mL}^{-1}\end{array}$ & $\begin{array}{l}\text { whole } \\
\text { mouse } \\
\text { serum. }\end{array}$ & ---- & $\begin{array}{c}30 \text { min } \\
\text { (incubation } \\
\text { time) }\end{array}$ \\
\hline 17 & Immunosensor & $\begin{array}{l}\text { Electrochemical } \\
\text { Impedance } \\
\text { spectroscopy }\end{array}$ & gold electrode & IL-6 & $\begin{array}{c}220 \\
\mathrm{pg} \\
\mathrm{mL}^{-1}\end{array}$ & PBS & --- & --- & --- & $\begin{array}{c}60 \text { min } \\
\text { (incubation } \\
\text { time) } 10 \\
\text { seconds } \\
\text { signal }\end{array}$ \\
\hline 18 & Aptamers & Conductance & carbon nanotube & IL-6 & $\begin{array}{l}1 \mathrm{pg} \\
\mathrm{mL}^{-1}\end{array}$ & PBS & $\begin{array}{l}1 \mathrm{pg} \mathrm{mL}^{-1}- \\
10 \mathrm{ng} \mathrm{mL}^{-1}\end{array}$ & blood & $10 \mathrm{pg} / \mathrm{mL}$ & real-time \\
\hline 19 & $\begin{array}{l}\text { Immunosensor } \\
\text { and aptamer }\end{array}$ & Electrochemical & $\begin{array}{c}\text { Organic Field Effect } \\
\text { Transistors }\end{array}$ & IL-6 & $\begin{array}{c}20 \mathrm{pg} \\
\mathrm{mL}^{-1}\end{array}$ & PBS & $\begin{array}{c}1 \mathrm{pM}-10 \\
\mathrm{nM}\end{array}$ & ---- & ---- & ---- \\
\hline 20 & Immunosensor & ELISA & $\begin{array}{l}\text { ultrafiltration } \\
\text { regenerated }\end{array}$ & IL-6 & $\begin{array}{l}31 \mathrm{pg} \\
\mathrm{mL}^{-1}\end{array}$ & PBS & $\begin{array}{l}31-500 \mathrm{pg} \\
\mathrm{mL}^{-1}\end{array}$ & ----- & ----- & $2 \mathrm{~h}$ \\
\hline
\end{tabular}




\begin{tabular}{|c|c|c|c|c|c|c|c|c|c|c|}
\hline & & & $\begin{array}{c}\text { cellulose } \\
\text { membranes } \\
(\mathrm{RC})\end{array}$ & & & & & & & \\
\hline 21 & Immunosensor & $\begin{array}{l}\text { Naked eye } \\
\text { and } \\
\text { optical } \\
\text { spectroscopy }\end{array}$ & $\begin{array}{c}\text { magnetic } \\
\text { nanoparticles } \\
\text { (MNPs) and } \\
\text { polystyrene } \\
\text { (PS) microparticles }\end{array}$ & IL-6 & $\begin{array}{c}11 \mathrm{pg} \\
\mathrm{mL}^{-1} \\
\text { eye } \\
1.2 \\
\mathrm{pg} \\
\mathrm{mL}^{-1} \\
\text { instr }\end{array}$ & PBS & $\begin{array}{c}3.7-900 \\
\mathrm{pg} \mathrm{m} \mathrm{m}^{-1} \\
\text { instrument }\end{array}$ & $\begin{array}{l}\text { human } \\
\text { serum }\end{array}$ & $\begin{array}{l}4,15 \mathrm{pg} / \mathrm{ml} \\
\text { instrument }\end{array}$ & $1 \mathrm{~h}$ \\
\hline 22 & Immunosensor & Chemiluminescence & $\begin{array}{c}\text { Polydimethylsiloxane } \\
\text { (PDMS) }\end{array}$ & IL-6 & $\begin{array}{c}5- \\
1280 \\
\mathrm{pg} \\
\mathrm{mL}^{-1}\end{array}$ & $\begin{array}{l}\text { PBS with } \\
30 \% \text { fetal calf } \\
\text { serum }\end{array}$ & $\begin{array}{l}5-1280 \\
p g ~ m L^{-1}\end{array}$ & $\begin{array}{l}\text { human } \\
\text { serum }\end{array}$ & --- & $90 \mathrm{~min}$ \\
\hline 23 & Immunosensor & Electrochemical & carbon electrode & IFN-Y & $\begin{array}{c}1.6 \mathrm{pg} \\
\mathrm{mL}^{-1}\end{array}$ & PBS & $\begin{array}{c}2.5-2000 \\
p g \mathrm{~mL}^{-1}\end{array}$ & saliva & --- & $>125 \min$ \\
\hline 24 & Immunosensor & Electrochemical & graphene & $\begin{array}{l}\text { IFN-y and } \\
\text { IL-10 }\end{array}$ & $\begin{array}{c}\text { IFN-Y: } \\
25 \mathrm{pg} \\
\mathrm{mL}^{-1} \\
\text { and } \\
\mathrm{IL}-10: \\
46 \mathrm{pg} \\
\mathrm{mL}^{-1}\end{array}$ & $\begin{array}{l}\text { Bovine } \\
\text { implant } \\
\text { serum }\end{array}$ & $\begin{array}{c}\text { IFN-Y: } \\
0.1-5 \mathrm{ng} \\
\mathrm{mL}^{-1} ; \mathrm{IL}- \\
10: 0.1-2 \\
\mathrm{ng} \mathrm{mL}\end{array}$ & --- & --- & $30 \mathrm{~min}$ \\
\hline 25 & Immunosensor & $\begin{array}{l}\text { Capacitance } \\
\text { electrochemical }\end{array}$ & silicon nitride & $\begin{array}{l}\text { Tumour } \\
\text { Necrosis } \\
\text { Factor } \\
\text { Alpha } \\
\text { (TNF- } \alpha) \\
\end{array}$ & $\begin{array}{l}0.38 \\
\mathrm{pg} \\
\mathrm{mL}^{-1}\end{array}$ & PBS & $\begin{array}{l}1 \mathrm{pg} \mathrm{mL}^{-1}- \\
30 \mathrm{pg} \mathrm{mL}^{-1}\end{array}$ & $\begin{array}{l}\text { artificial } \\
\text { saliva }\end{array}$ & $1 \mathrm{pg} / \mathrm{mL}$ & $90 \mathrm{~min}$ \\
\hline 26 & Immunosensor & $\begin{array}{l}\text { Electrochemical } \\
\text { impedance }\end{array}$ & $\begin{array}{c}\text { Polyaniline } \\
\text { deposited } \\
\text { on a graphene } \\
\text { screen-printed } \\
\text { paper electrode }\end{array}$ & IFN-Y & $\begin{array}{c}3.4 \mathrm{pg} \\
\mathrm{mL}^{-1}\end{array}$ & PBS & $\begin{array}{l}5-1000 \\
p g \mathrm{~mL}^{-1}\end{array}$ & $\begin{array}{c}\text { purified } \\
\text { human } \\
\text { serum }\end{array}$ & --- & $30 \mathrm{~min}$ \\
\hline 27 & Immunosensor & Electrochemical & $\begin{array}{c}\text { magnetic beads } \\
\text { and } \\
\text { gold } \\
\text { electrodes }\end{array}$ & IL-3 & --- & PBS & --- & plasma & $5 \mathrm{pg} / \mathrm{mL}$ & $60 \mathrm{~min}$ \\
\hline
\end{tabular}




\begin{tabular}{|c|c|c|c|c|c|c|c|c|c|c|}
\hline 28 & Immunosensor & Electrochemical & gold electrodes & TNF- $\alpha$ & $\begin{array}{l}1 \mathrm{pg} \\
\mathrm{mL}^{-1}\end{array}$ & PBS & $\begin{array}{c}1-30 \mathrm{pg} \\
\mathrm{mL}^{-1}\end{array}$ & $\begin{array}{c}\text { human } \\
\text { saliva }\end{array}$ & --- & $35 \min$ \\
\hline 29 & Immunosensor & Photoelectrochemical & $\begin{array}{c}\mathrm{CdS} \text { and } \mathrm{Mn} \\
\text { cosensitized } \mathrm{TiO}_{2} \\
\text { nanorod arrays } \\
\text { electrode }\end{array}$ & TNF- $\alpha$ & $\begin{array}{l}1.0 \mathrm{pg} \\
\mathrm{mL}^{-1}\end{array}$ & PBS & $\begin{array}{c}2.0 \mathrm{pg} \mathrm{mL}^{-} \\
1-200 \mathrm{ng} \\
\mathrm{mL}^{-1}\end{array}$ & $\begin{array}{l}\text { human } \\
\text { serum }\end{array}$ & --- & $120 \min$ \\
\hline 30 & Immunosensor & $\begin{array}{l}\text { Electrochemical } \\
\text { impedance }\end{array}$ & $\begin{array}{c}\text { reduced graphene } \\
\text { oxide } \\
\text { (RGO) coated with } \\
\text { AuNP on an indium } \\
\text { tin oxide } \\
\text { microelectrode } \\
\text { array } \\
\end{array}$ & TNF- $\alpha$ & $\begin{array}{l}0.43 \\
\mathrm{pg} \\
\mathrm{mL}^{-1}\end{array}$ & PBS & $\underset{\mathrm{mL}^{-1}}{1-1000 \mathrm{pg}}$ & --- & --- & --- \\
\hline 31 & Immunosensor & Electrochemical & $\begin{array}{l}\text { PPC-PBA mixed } \\
\text { layers on ITO }\end{array}$ & TNF- $\alpha$ & $\begin{array}{l}10 \mathrm{pg} \\
\mathrm{mL}^{-1}\end{array}$ & $\begin{array}{c}\text { PBS + } \\
\text { hemoglobin } \\
(5 \mu \mathrm{g} / \mathrm{mL}) \text { and } \\
\text { human } \\
\text { serum } \\
\text { albumin. }\end{array}$ & $\begin{array}{c}0.01 \\
\mathrm{ng} \mathrm{mL} \mathrm{m}^{-1}- \\
500 \mathrm{ng} \\
\mathrm{mL}^{-1}\end{array}$ & $\begin{array}{l}\text { whole } \\
\text { blood }\end{array}$ & --- & $>60 \min$ \\
\hline 32 & Aptasensor & Electrochemical & Magnetic nanobeads & IFN- $\gamma$ & $\begin{array}{l}6 \mathrm{pg} \\
\mathrm{mL}^{-1}\end{array}$ & $\begin{array}{l}\mathrm{pH} 7.5, \text { salt } \\
\text { concentration } \\
\text { of } 100 \mathrm{mM}\end{array}$ & $\begin{array}{c}10-500 \mathrm{pg} \\
\mathrm{mL}^{-1}\end{array}$ & serum & --- & ---- \\
\hline
\end{tabular}

1) Nie, R.; Huang, J.; Xu, X.; Yang, L. A Portable Pencil-like Immunosensor for Point-of-Care Testing of Inflammatory Biomarkers. Anal. Bioanal. Chem. 2020, 412 (13), 3231-3239. https://doi.org/10.1007/s00216-020-02582-z.

(2) Aydın, E. B. Highly Sensitive Impedimetric Immunosensor for Determination of Interleukin 6 as a Cancer Biomarker by Using Conjugated Polymer Containing Epoxy Side Groups Modified Disposable ITO Electrode. Talanta 2020, 215, 120909.

https://doi.org/10.1016/j.talanta.2020.120909.

(3) Alba-Patiño, A.; Russell, S. M.; Borges, M.; Pazos-Pérez, N.; Álvarez-Puebla, R. A.; De La Rica, R. Nanoparticle-Based Mobile Biosensors for the Rapid Detection of Sepsis Biomarkers in Whole Blood. Nanoscale Adv. 2020, 2 (3), $1253-1260$.

https://doi.org/10.1039/d0na00026d. 
(4) Sabaté del Río, J.; Henry, O. Y. F.; Jolly, P.; Ingber, D. E. An Antifouling Coating That Enables Affinity-Based Electrochemical Biosensing in Complex Biological Fluids. Nat. Nanotechnol. 2019, 14 (12), 1143-1149. https://doi.org/10.1038/s41565-019-0566-z.

(5) Fabri-Faja, N.; Calvo-Lozano, O.; Dey, P.; Terborg, R. A.; Estevez, M. C.; Belushkin, A.; Yesilköy, F.; Duempelmann, L.; Altug, H.; Pruneri, V.; Lechuga, L. M. Early Sepsis Diagnosis via Protein and MiRNA Biomarkers Using a Novel Point-of-Care Photonic Biosensor. Anal. Chim. Acta 2019, 1077, 232-242. https://doi.org/10.1016/j.aca.2019.05.038.

(6) Wang, Y.; Sun, J.; Hou, Y.; Zhang, C.; Li, D.; Li, H.; Yang, M.; Fan, C.; Sun, B. A SERS-Based Lateral Flow Assay Biosensor for Quantitative and Ultrasensitive Detection of Interleukin-6 in Unprocessed Whole Blood. Biosens. Bioelectron. 2019, $141,111432$. https://doi.org/10.1016/j.bios.2019.111432.

(7) Tertis, M.; Leva, P. I.; Bogdan, D.; Suciu, M.; Graur, F.; Cristea, C. Impedimetric Aptasensor for the Label-Free and Selective Detection of Interleukin-6 for Colorectal Cancer Screening. Biosens. Bioelectron. 2019, 137, 123-132. https://doi.org/10.1016/j.bios.2019.05.012.

(8) Tertiş, M.; Melinte, G.; Ciui, B.; Şimon, I.; Ştiufiuc, R.; Săndulescu, R.; Cristea, C. A Novel Label Free Electrochemical Magnetoimmunosensor for Human Interleukin-6 Quantification in Serum. Electroanalysis 2019, 31 (2), 282-292. https://doi.org/10.1002/elan.201800620.

(9) Huang, D.; Ying, H.; Jiang, D.; Liu, F.; Tian, Y.; Du, C.; Zhang, L.; Pu, X. Rapid and Sensitive Detection of Interleukin-6 in Serum via Time-Resolved Lateral Flow Immunoassay. Anal. Biochem. 2020, 588, 113468. https://doi.org/10.1016/j.ab.2019.113468.

(10) Borse, V.; Srivastava, R. Fluorescence Lateral Flow Immunoassay Based Point-of-Care Nanodiagnostics for Orthopedic ImplantAssociated Infection. Sensors Actuators, B Chem. 2019, 280, 24-33. https://doi.org/10.1016/j.snb.2018.10.034.

(11) Hao, Z.; Pan, Y.; Shao, W.; Lin, Q.; Zhao, X. Graphene-Based Fully Integrated Portable Nanosensing System for on-Line Detection of Cytokine Biomarkers in Saliva. Biosens. Bioelectron. 2019, 134, 16-23. https://doi.org/10.1016/j.bios.2019.03.053.

(12) Russell, C.; Ward, A. C.; Vezza, V.; Hoskisson, P.; Alcorn, D.; Steenson, D. P.; Corrigan, D. K. Development of a Needle Shaped Microelectrode for Electrochemical Detection of the Sepsis Biomarker Interleukin-6 (IL-6) in Real Time. Biosens. Bioelectron. 2019, 126, 806814. https://doi.org/10.1016/j.bios.2018.11.053. 
(13) Zhu, J.; He, J.; Verano, M.; Brimmo, A. T.; Glia, A.; Qasaimeh, M. A.; Chen, P.; Aleman, J. O.; Chen, W. An Integrated AdiposeTissue-on-Chip Nanoplasmonic Biosensing Platform for Investigating Obesity-Associated Inflammation. Lab Chip 2018, 18 (23), 3550-3560. https://doi.org/10.1039/c81c00605a.

(14) Chen, H.; Li, J.; Zhang, X.; Li, X.; Yao, M.; Zheng, G. Automated in Vivo Nanosensing of Breath-Borne Protein Biomarkers. Nano Lett. 2018, 18 (8), 4716-4726. https://doi.org/10.1021/acs.nanolett.8b01070.

(15) Zhang, K.; Liu, G.; Goldys, E. M. Robust Immunosensing System Based on Biotin-Streptavidin Coupling for Spatially Localized Femtogram ML-1 Level Detection of Interleukin-6. Biosens. Bioelectron. 2018, 102, 80-86. https://doi.org/10.1016/j.bios.2017.11.023. (16) Wei, H.; Ni, S.; Cao, C.; Yang, G.; Liu, G. Graphene Oxide Signal Reporter Based Multifunctional Immunosensing Platform for Amperometric Profiling of Multiple Cytokines in Serum. ACS Sensors 2018, 3 (8), 1553-1561. https://doi.org/10.1021/acssensors.8b00365. (17) Gentili, D.; D’Angelo, P.; Militano, F.; Mazzei, R.; Poerio, T.; Brucale, M.; Tarabella, G.; Bonetti, S.; Marasso, S. L.; Cocuzza, M.; Giorno, L.; Iannotta, S.; Cavallini, M. Integration of Organic Electrochemical Transistors and Immuno-Affinity Membranes for Label-Free Detection of Interleukin-6 in the Physiological Concentration Range through Antibody-Antigen Recognition. J. Mater. Chem. B 2018, 6 (33), 5400-5406. https://doi.org/10.1039/c8tb01697f.

(18) Khosravi, F.; Loeian, S.; Panchapakesan, B. Ultrasensitive Label-Free Sensing of IL-6 Based on PASE Functionalized Carbon Nanotube Micro-Arrays with RNA-Aptamers as Molecular Recognition Elements. Biosensors 2017, 7 (4), 17. https://doi.org/10.3390/bios7020017.

(19) Diacci, C.; Berto, M.; Di Lauro, M.; Bianchini, E.; Pinti, M.; Simon, D. T.; Biscarini, F.; Bortolotti, C. A. Label-Free Detection of Interleukin-6 Using Electrolyte Gated Organic Field Effect Transistors. Biointerphases 2017, 12 (5), 05F401. https://doi.org/10.1116/1.4997760. (20) Militano, F.; Poerio, T.; Mazzei, R.; Salerno, S.; Bartolo, L. De; Giorno, L. Development of Biohybrid Immuno-Selective Membranes for Target Antigen Recognition. Biosens. Bioelectron. 2017, 92, 54-60. https://doi.org/10.1016/j.bios.2017.02.003.

(21) Yin, B.; Zheng, W.; Dong, M.; Yu, W.; Chen, Y.; Joo, S. W.; Jiang, X. An Enzyme-Mediated Competitive Colorimetric Sensor Based on Au@Ag Bimetallic Nanoparticles for Highly Sensitive Detection of Disease Biomarkers. Analyst 2017, 142 (16), $2954-2960$. https://doi.org/10.1039/c7an00779e. 
(22) Wu, J.; Chen, Y.; Yang, M.; Wang, Y.; Zhang, C.; Yang, M.; Sun, J.; Xie, M.; Jiang, X. Streptavidin-Biotin-Peroxidase NanocomplexAmplified Microfluidics Immunoassays for Simultaneous Detection of Inflammatory Biomarkers. Anal. Chim. Acta 2017, 982, $138-147$. https://doi.org/10.1016/j.aca.2017.05.031.

(23) Sánchez-Tirado, E.; González-Cortés, A.; Yáñez-Sedeño, P.; Pingarrón, J. M. Electrochemical Immunosensor for the Determination of the Cytokine Interferon Gamma (IFN- $\gamma$ ) in Saliva. Talanta 2020, 211, 120761. https://doi.org/10.1016/j.talanta.2020.120761.

(24) Parate, K.; Rangnekar, S. V.; Jing, D.; Mendivelso-Perez, D. L.; Ding, S.; Secor, E. B.; Smith, E. A.; Hostetter, J. M.; Hersam, M. C.; Claussen, J. C. Aerosol-Jet-Printed Graphene Immunosensor for Label-Free Cytokine Monitoring in Serum. ACS Appl. Mater. Interfaces 2020, 12 (7), 8592-8603. https://doi.org/10.1021/acsami.9b22183.

(25) Bahri, M.; Baraket, A.; Zine, N.; Ben Ali, M.; Bausells, J.; Errachid, A. Capacitance Electrochemical Biosensor Based on Silicon Nitride Transducer for TNF- $\alpha$ Cytokine Detection in Artificial Human Saliva: Heart Failure (HF). Talanta 2020, 209, 120501.

https://doi.org/10.1016/j.talanta.2019.120501.

(26) Ruecha, N.; Shin, K.; Chailapakul, O.; Rodthongkum, N. Label-Free Paper-Based Electrochemical Impedance Immunosensor for Human Interferon Gamma Detection. Sensors Actuators, B Chem. 2019, 279, 298-304. https://doi.org/10.1016/j.snb.2018.10.024.

(27) Min, J.; Nothing, M.; Coble, B.; Zheng, H.; Park, J.; Im, H.; Weber, G. F.; Castro, C. M.; Swirski, F. K.; Weissleder, R.; Lee, H. Integrated Biosensor for Rapid and Point-of-Care Sepsis Diagnosis. ACS Nano 2018, 12 (4), 3378-3384.

https://doi.org/10.1021/acsnano.7b08965.

(28) Barhoumi, L.; Baraket, A.; Bellagambi, F. G.; Karanasiou, G. S.; Ali, M. Ben; Fotiadis, D. I.; Bausells, J.; Zine, N.; Sigaud, M.; Errachid, A. A Novel Chronoamperometric Immunosensor for Rapid Detection of TNF-A in Human Saliva. Sensors Actuators, B Chem. 2018, 266, 477-484. https://doi.org/10.1016/j.snb.2018.03.135.

(29) Liu, A.; Shan, H.; Ma, M.; Shangguan, L.; Jiang, K.; Shi, M.; Zhao, Y.; Liu, S.; Li, S. An Ultrasensitive Photoelectrochemical Immunosensor by Integration of Nanobody, TiO2 Nanorod Arrays and ZnS Nanoparticles for the Detection of Tumor Necrosis Factor- $\alpha$. J. Electroanal. Chem. 2017, 803, 1-10. https://doi.org/10.1016/j.jelechem.2017.09.008. 
(30) Yagati, A. K.; Lee, G. Y.; Ha, S.; Chang, K. A.; Pyun, J. C.; Cho, S. Impedimetric Tumor Necrosis Factor- $\alpha$ Sensor Based on a Reduced Graphene Oxide Nanoparticle-Modified Electrode Array. J. Nanosci. Nanotechnol. 2016, 16 (11), 11921-11927. https://doi.org/10.1166/jnn.2016.13618.

(31) Jiang, C.; Alam, M. T.; Silva, S. M.; Taufik, S.; Fan, S.; Gooding, J. J. Unique Sensing Interface That Allows the Development of an Electrochemical Immunosensor for the Detection of Tumor Necrosis Factor $\alpha$ in Whole Blood. ACS Sensors 2016, 1 (12), $1432-1438$. https://doi.org/10.1021/acssensors.6b00532.

(32) Guozhen Liu, Chaomin Cao, Shengnan N, Shilun Feng, and Hui Wei. On-chip structure-switching aptamer-modified magnetic nanobeads for the continuous monitoring of interferon-gamma ex vivo. Microsystems \& Nanoengineering (2019) 5:35 